\title{
FIGO Stage IIA1
}

National Cancer Institute

\section{Source}

National Cancer Institute. FIGO Stage IIA1. NCI Thesaurus. Code C162223.

A FIGO stage term that applies to gynecologic cancers. For cervical cancer, it refers to cancer limited to the upper two-thirds of the vagina without parametrial involvement, less than $4 \mathrm{~cm}$ in greatest dimension. 